\title{
Correlation Between Montreal Cognitive Assessment and Functional Outcome in Subacute Stroke Patients With Cognitive Dysfunction
}

\author{
Kil-Byung Lim, MD, Jiyong Kim, MD, Hong-Jae Lee, MD, JeeHyun Yoo, MD, \\ Eun-Cheol You, MD, Joongmo Kang, MD
}

Department of Physical Medicine and Rehabilitation, Inje University Ilsan Paik Hospital, Goyang, Korea

Objective To investigate the correlation between the Montreal Cognitive Assessment (MoCA) and functional outcome among subacute stroke patients with cognitive dysfunction.

Methods Records of 61 inpatients were reviewed. Patients were divided into two groups based on their initial MoCA score. MoCA score of 11 was set as the differentiating criterion. We compared the improvements in Modified Barthel Index (MBI) from initial assessment to discharge between the two groups.

Results There were no significant differences between the two groups in relation to age, duration from onset to admission, hospitalization period, or years of education. In a comparison of the results of Mini-Mental Status Examinations (MMSE) administered at admission and again at discharge, there was significantly more improvement in MMSE scores in the group with low MoCA scores than in the group with high MoCA scores. However, the group with high MoCA scores also showed high MBI scores at discharge and exhibited greater MBI improvement.

Conclusion Higher initial MoCA scores (which reflect preservation of executive function) indicate better functional outcome in the subacute stroke phase.

Keywords Stroke, Cognitive dysfunction, Activities of daily living, Executive function

Received May 19, 2017; Accepted July 11, 2017

Corresponding author: Eun-Cheol You

Department of Physical Medicine and Rehabilitation, Inje University Ilsan Paik Hospital, 170 Juhwa-ro, Ilsanseo-gu, Goyang 10380, Korea. Tel: +82-31910-7440, Fax: +82-31-910-7446, E-mail: 19684@paik.ac.kr

ORCID: Kil-Byung Lim (http://orcid.org/0000-0001-8971-7486); Jiyong Kim (http://orcid.org/0000-0003-4693-8400); Hong-Jae Lee (http://orcid. org/0000-0001-9813-4952); JeeHyun Yoo (http://orcid.org/0000-0002-1555-8029); Eun-Cheol You (http://orcid.org/0000-0002-6435-7813); Joongmo Kang (http://orcid.org/0000-0003-3133-9202).

(c) This is an open-access article distributed under the terms of the Creative Commons Attribution Non-Commercial License (http://creativecommons.org/ licenses/by-nc/4.0) which permits unrestricted noncommercial use, distribution, and reproduction in any medium, provided the original work is properly cited. Copyright $\odot 2018$ by Korean Academy of Rehabilitation Medicine 


\section{INTRODUCTION}

Seventy-five percent of stroke survivors experience mobility difficulties, fatigue, emotional distress, and cognitive impairment [1]. Cognitive and motor impairments are of particular interest, as these two factors may influence the recovery of functional status during rehabilitation. Problems in the two areas prolong rehabilitation, undermine the ability to independently engage in daily activities, and diminish quality of life [2].

The primary goal of stroke patients' treatment is to resolve these problems and to enhance survivors' independence. In other words, the objective of rehabilitation is to enhance functional independence of daily activities and help survivors return to a fully functioning lifestyle [3].

Identified predictors that define functionality following a stroke include the patient's age, the stroke mechanism, the location and volume of lesions, and comorbidities [4]. The relationship between cognitive function and functional status has been studied recently by comparatively analyzing patients with normal cognitive function and patients with decreased cognitive function [5]. The results of these studies indicate that cognitive abilities have functional implications and can predict functional outcomes in stroke and various other diseases. Cognitive dysfunction is a common complication of stroke, affecting $35 \%$ of all stroke survivors and $31 \%$ to $91 \%$ in acute and subacute stroke patients $[6,7]$. Cognitive dysfunction such as decreased memory and executive function, dyspraxia, and lack of attention are common in stroke patients $[8,9]$. Studies have been conducted on the relationship between initial post-stroke cognitive function and functional prognosis [10,11].

The Mini-Mental Status Examination (MMSE) and the Montreal Cognitive Assessment (MoCA) are two representative tools for assessing general cognitive function. However, most previous studies used MMSE to examine the relationship between cognitive function and functional outcomes in stroke patients $[12,13]$.

MMSE is the most commonly used screening test for cognitive evaluation, but MMSE is not sensitive enough to find cognitive impairments in stroke patients nor is it able to precisely identify the domain and severity of cognitive defects [14]. MMSE's limited ability to distinguish mild cognitive impairment (MCI) is well documented and can be attributed to lack of complexity and execu- tive function items (including inhibitory control, working memory, and cognitive flexibility) [15].

Conversely, MoCA was found to be more sensitive to detecting cognitive dysfunction [16]. Furthermore, MMSE was, in fact, initially designed to assess psychiatric disorders; as such, MoCA is gaining prevalence in assessing dementia. However, existing studies on MoCA and functional outcome were mostly performed on elderly patients with heterogeneous diseases and mild functional impairment. Durant et al. [17] found that lower a MoCA score was associated with greater functional impairment in neurodegenerative diseases, including Alzheimer disease, mild cognitive impairment, cognitive disorder, Lewy body dementia, vascular dementia, etc. However, these studies had the limitation of being a heterogeneous clinical sample, focusing on current function rather than the recovery of the patient's function. The results of the current study, though it specifically targeted stroke patients, were similar to those of existing neurodegenerative diseases.

Recently, studies by Toglia et al. [18] have shown that MoCA shows stronger correlation to functional outcome in patients with subacute stroke than MMSE does. The studies have demonstrated that MoCA has less ceiling effect and stronger internal reliability than MMSE. However, the results of the study cannot be applied to the entire stroke population; the subjects of the study exhibited very mild cognitive deficits (mean MMSE score, 25). In addition, the sample primarily comprised Caucasian males with a high level of education [18].

Therefore, the aim of this study is to investigate the relationship between MoCA and functional outcome in subacute stroke patients with mild to moderate/severe cognitive deficit and broad levels of education.

\section{MATERIALS AND METHODS}

\section{Study subjects}

The study was conducted using records of 212 patients diagnosed with cerebrovascular diseases admitted to the Department of Physical Medicine and Rehabilitation, Inje University Ilsan Paik Hospital. The data accumulation period was January 2014 to February 2017. Of the 212 patients, 61 were recruited for eligibility screening. The group consisted of 36 patients with right hemisphere damage (RHD) and 25 patients with left hemisphere 
damage (LHD).

The inclusion criteria were as follows: (1) 'probable vascular dementia' diagnosed in accordance with the NINDS-AIREN criteria [19], (2) cerebrovascular disease only in the right or left hemisphere (e.g., pons, midbrain, and cerebellum lesions are excluded), (3) MMSE score of $\leq 26$, and (4) Clinical Dementia Rating scale (CDR) 0.5 to $2[20,21]$.

The exclusion criteria were (1) major disability (modified Rankin Scale [mRS] >4), (2) significant aphasia or dysarthria that impeded cognitive assessment, (3) major and active psychiatric illness and pre-existing dementia/ previous stroke history, and (4) presence of cerebrovascular disease in both hemispheres. All patients received conventional physical and occupational therapy five times per week (Fig. 1).

\section{Measures}

On the first day of hospitalization, subjects underwent medical-history submission, physical examination, activities of daily living (ADL) evaluation using Modified Barthel Index (MBI), and cognition evaluation using the Korean versions of the MMSE (K-MMSE) and the MoCA (K-MoCA, K2-Chuncheon). MoCA is a cognitive screening tool requiring approximately 10 minutes to administer (Fig. 2). It can evaluate frontal lobe executive function and abstractive function that cannot be evaluated by conventional cognitive testing tools [22]. Since K-MoCA differentiates cognition levels in patients with mild dementia from those with severe dementia, K-MoCA is more sensitive than K-MMSE in detecting MCI [23]. The test can be administered by various levels of health care providers. MoCA consists of 12 individual tasks grouped into cognitive domains including (1) visuospatial/executive functioning, (2) naming, (3) attention, (4) language, (5) abstraction, (6) memory, and (7) orientation. Educational correction is applicable (i.e., one point added for individuals with 12 years of education or less) in total score calculation. A total score of $\leq 26$ on MoCA indicates cognitive impairment, according to clinical standards. One day before the patients were discharged, K-MMSE and MBI were tested.

\section{Patient classification}

\section{Degree of severity}

The severity of a patient's cognitive impairment was defined by score intervals on the MMSE [24] and MMSEMoCA conversion table [25]. MMSE scoring $\geq 20$ were considered as mild dementia, while patients scoring between 10 and 19 were classified as having moderate dementia. Patients scoring $\leq 9$ were classified as suffering from severe dementia [24].

A MoCA score of 11 equals 20 on MMSE, according to the MMSE-MoCA conversion table created by Bergeron et al. [25]. On this basis, a MoCA score of 11 was set as the criterion for dividing the mild and moderate/severe groups.

Patients with a MoCA score of 11 or higher were classified as mild and those with a score of less than 11 were classified as moderate/severe.

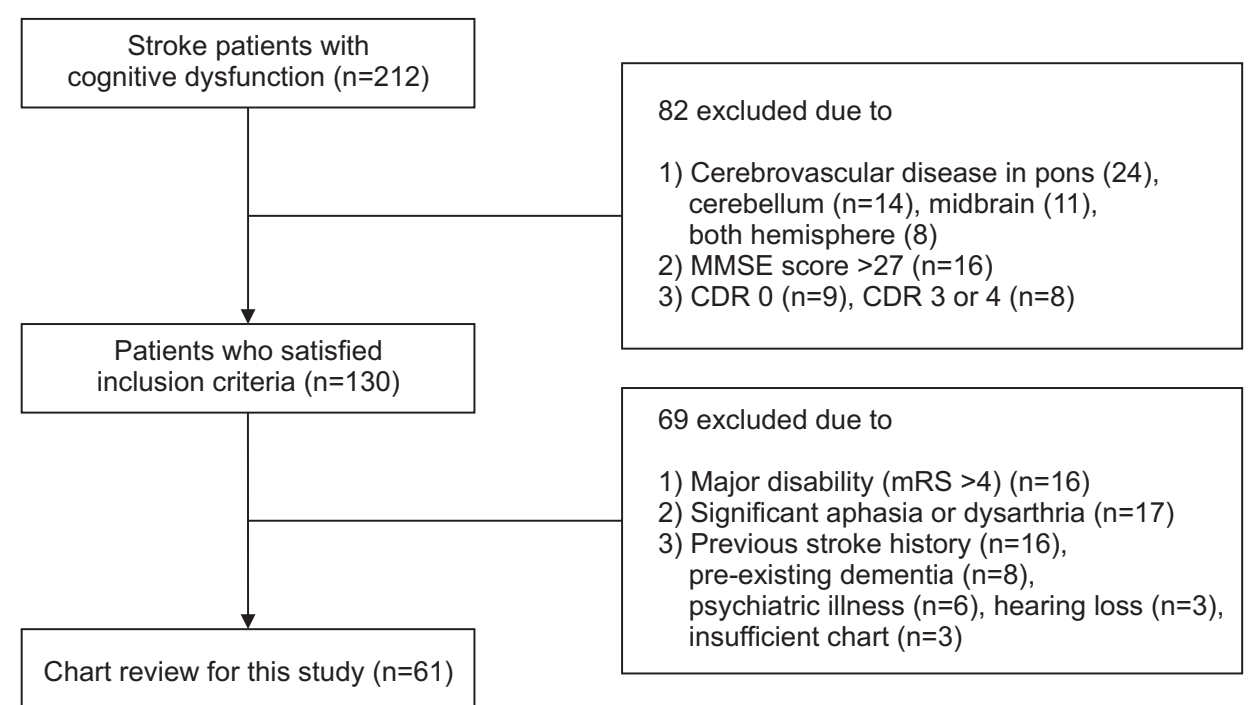

Fig. 1. Flow chart for enrolling subjects. MMSE, Mini-Mental Status Examination; CDR, Clinical Dementia Rating; mRS, modified Rankin Scale. 


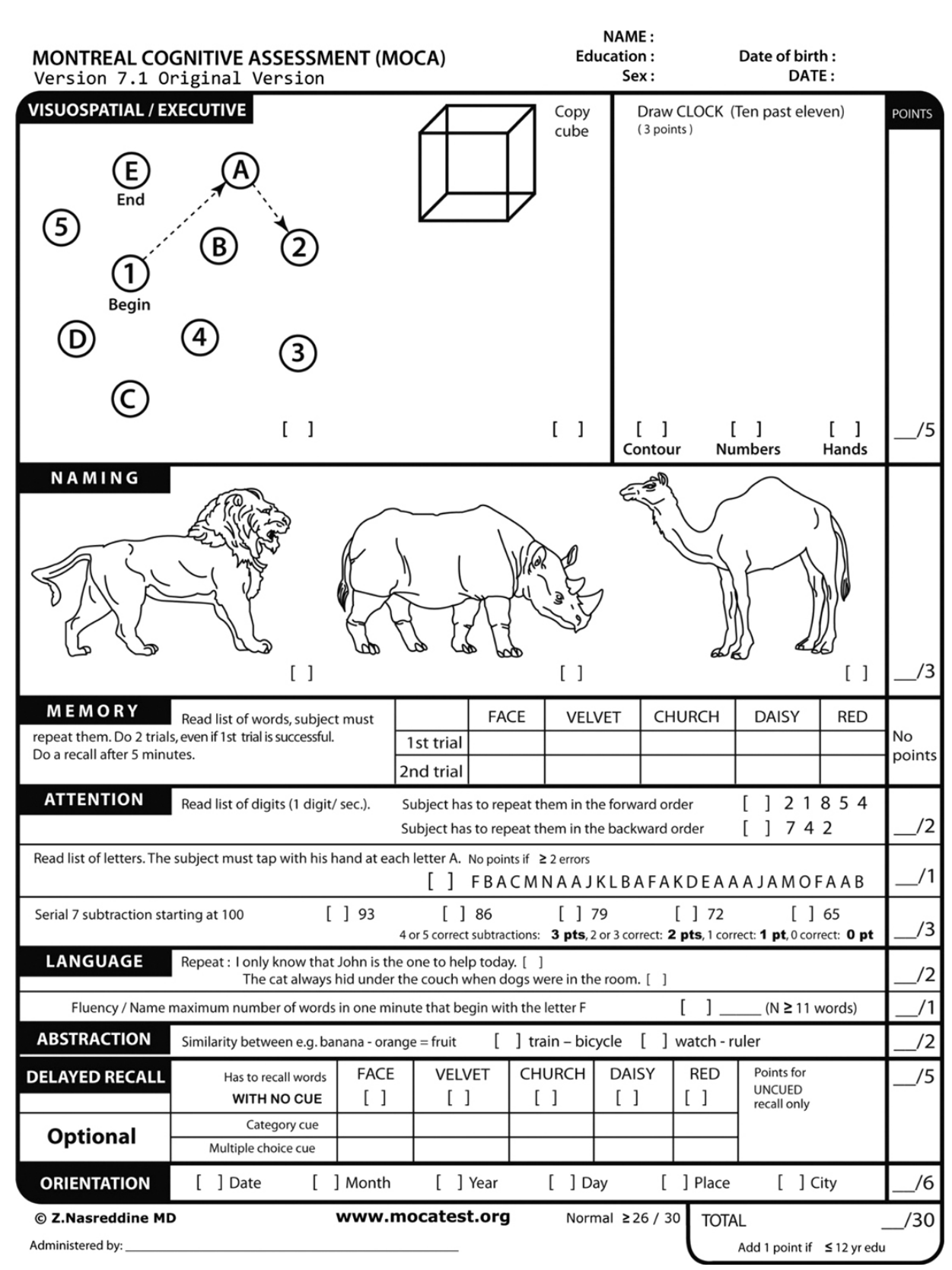

Fig. 2. Montreal Cognitive Assessment (MoCA).

\section{Analyses}

IBM's statistical-analysis platform, SPSS version 21.0 (IBM SPSS, Armonk, NY, USA), was used for statistical analyses. Wilcoxon signed-rank test was performed to compare the difference in MBI and the degree of MBI improvement between two groups, and the changes in MBI and sub-items were compared using the Mann-Whitney test. Statistical significance was recognized when p-value was less than 0.05 .

In addition, linear regression analysis was performed to confirm the correlation between cognitive function test tools (K-MMSE, K-MoCA) and functional outcomes (follow-up MBI score, MBI improvement).

\section{RESULTS}

Following the inclusion and exclusion criteria of the study, 61 charts of 212 patients were reviewed.

\section{General characteristics of patients}

General characteristics of the patients are listed in Table 1. According to the MoCA score, 31 patients were catego- 
Table 1. General characteristics of patients

\begin{tabular}{|c|c|c|c|}
\hline & $\begin{array}{c}\text { Group A } \\
(\mathrm{MoCA}<\mathbf{1 1}) \\
(\mathbf{n}=\mathbf{3 0})\end{array}$ & $\begin{array}{c}\text { Group B } \\
(M o C A \geq 11) \\
(n=31)\end{array}$ & $\begin{array}{l}\text { p-value } \\
\text { (group } \\
\text { A vs. B) } \\
\end{array}$ \\
\hline Mean MoCA & $5.66 \pm 3.14$ & $14.48 \pm 3.13$ & \\
\hline \multicolumn{4}{|l|}{ Sex } \\
\hline Male & 17 & 18 & \\
\hline Female & 13 & 13 & \\
\hline \multicolumn{4}{|l|}{ Side of stroke } \\
\hline Right & 11 & 16 & \\
\hline Left & 19 & 15 & \\
\hline \multicolumn{4}{|l|}{ Type of stroke } \\
\hline Hemorrhagic & 18 & 18 & \\
\hline Ischemic & 12 & 13 & \\
\hline Age (yr) & $71.33 \pm 8.26$ & $69.65 \pm 6.72$ & 0.384 \\
\hline Time post stroke (day) & $22.93 \pm 12.01$ & $21.19 \pm 9.90$ & 0.539 \\
\hline Stay length (day) & $24.10 \pm 7.51$ & $24.74 \pm 10.21$ & 0.781 \\
\hline Education (year) & $10.20 \pm 4.44$ & $10.77 \pm 3.19$ & 0.436 \\
\hline
\end{tabular}

Values are presented as mean \pm standard deviation.

MoCA, Montreal Cognitive Assessment.

${ }^{*} \mathrm{p}<0.05$.

rized in the mild group and 30 patients in the moderate/ severe group. There was no statistically significant difference between the two groups in terms of age, duration from onset to admission, hospitalization period, and level of education.

Improvement of cognitive deficit and functional impairment

The initial mean MMSE of all patients was $16.08 \pm 6.80$. The mean MBI score was $27.46 \pm 20.77$ and the mean CDR was $1.25 \pm 0.91$. The mean MMSE and MBI at the time of discharge were improved to $20.77 \pm 6.30$ and $45.47 \pm 23.58$.

The two groups, classified by MoCA score, showed statistically significant differences in initial MMSE and CDR, while the difference in initial MBI scores was not significant. However, the second test prior to discharge showed significant differences in MMSE and MBI between the two groups.

Comparing MMSE differences between initial assessment and discharge, the improvement of MMSE score was statistically greater in the group with low MoCA score $(5.47 \pm 3.43)$ than in the group with high MoCA score (3.94 \pm 2.67$)$, but the improvement of MBI score was greater in the high MoCA group $(26.03 \pm 15.29)$ than the
Table 2. Comparison of cognitive and functional outcome of patients

\begin{tabular}{|cccc}
\hline & $\begin{array}{c}\text { Group A } \\
(\mathbf{M o C A}<\mathbf{1 1}) \\
(\mathbf{n = 3 0})\end{array}$ & $\begin{array}{c}\text { Group B } \\
(\mathbf{M o C A} \geq \mathbf{1 1}) \\
(\mathbf{n = 3 1})\end{array}$ & $\begin{array}{c}\text { p-value } \\
(\text { group } \\
\text { A vs. B) }\end{array}$ \\
\hline Initial & & & \\
\hline MMSE & $11.50 \pm 6.62$ & $20.51 \pm 4.58$ & $<0.001^{*}$ \\
MBI & $26.30 \pm 21.77$ & $28.58 \pm 19.3$ & 0.667 \\
\hline CDR & $1.80 \pm 0.97$ & $0.91 \pm 0.55$ & $<0.001^{*}$ \\
Discharge & & & \\
MMSE & $16.97 \pm 6.88$ & $20.51 \pm 4.58$ & $<0.001^{*}$ \\
MBI & $36.27 \pm 24.76$ & $54.29 \pm 19.86$ & 0.003 \\
\hline Difference & & & \\
\hline MMSE & $5.47 \pm 3.43$ & $3.94 \pm 2.67$ & 0.056 \\
\hline MBI & $9.83 \pm 11.92$ & $26.03 \pm 15.29$ & $<0.001^{*}$ \\
\hline
\end{tabular}

Values are presented as mean \pm standard deviation.

MoCA, Montreal Cognitive Assessment; MMSE, MiniMental Status Examination; CDR, Clinical Dementia Rating; MBI, Modified Barthel Index.

${ }^{*} \mathrm{p}<0.05$.

low MoCA group (9.83 \pm 11.92$)$ (Table 2).

When comparing the initial and final MBI sub-items score of the two groups, all the final values were significantly improved over the initial scores. Comparing the initial and follow-up scores of each item of MBI in the two groups, all items except for bowel control change, ambulation, or wheelchair change showed a significant improvement in high MoCA group compared to low MoCA group (Table 3).

According to linear regression analysis, initial MMSE showed a significant positive correlation with followup MBI score $(\beta=0.619, t(95)=6.049, p<0.001)$ and initial K-MoCA showed significant positive correlation with follow-up MBI score $(\beta=0.564, t(95)=6.730, p<0.001)$. In addition, the initial MoCA score showed significant correlation with MBI improvement $(\beta=0.407, t(95)=4.393$, $\mathrm{p}<0.001)$. However, initial MMSE did not demonstrate significance related to MBI improvement $(\beta=0.184$, $t(95)=1.435, p=0.157)$. Age and level of education were controlled at the time of analysis.

\section{DISCUSSION}

In this study, both groups showed improvement in the MBI score when comparing initial and final values. MBI 
Table 3. Comparison of MBI and sub-item score

\begin{tabular}{|c|c|c|c|c|c|c|}
\hline & & $\begin{array}{c}\text { Group A } \\
(\text { MoCA }<11)\end{array}$ & p-value & $\begin{array}{c}\text { Group B } \\
(\text { MoCA } \geq 11)\end{array}$ & p-value & $\begin{array}{c}\text { p-value } \\
\text { (group A vs. B) }\end{array}$ \\
\hline \multirow[t]{3}{*}{ MBI score } & Initial & $26.27 \pm 21.73$ & $<0.001^{*}$ & $28.58 \pm 19.39$ & $<0.001^{*}$ & \\
\hline & Final & $36.23 \pm 24.74$ & & $54.29 \pm 19.86$ & & \\
\hline & Change & $10.07 \pm 11.81$ & & $25.70 \pm 14.20$ & & $<0.001^{*}$ \\
\hline \multirow[t]{3}{*}{ Personal hygiene } & Initial & $1.33 \pm 1.49$ & $<0.001^{*}$ & $1.84 \pm 1.44$ & $0.001^{*}$ & \\
\hline & Final & $1.90 \pm 1.56$ & & $3.48 \pm 1.06$ & & \\
\hline & Change & $0.67 \pm 0.88$ & & $1.65 \pm 1.11$ & & $<0.001^{*}$ \\
\hline \multirow[t]{3}{*}{ Bathing self } & Initial & $0.17 \pm 0.38$ & $<0.001^{*}$ & $0.45 \pm 0.93$ & $0.001^{*}$ & \\
\hline & Final & $0.77 \pm 1.04$ & & $2.16 \pm 1.66$ & & \\
\hline & Change & $0.60 \pm 0.93$ & & $1.71 \pm 1.55$ & & $0.001^{*}$ \\
\hline \multirow[t]{3}{*}{ Feeding } & Initial & $3.33 \pm 2.88$ & $<0.001^{*}$ & $4.00 \pm 2.91$ & $0.007^{*}$ & \\
\hline & Final & $4.20 \pm 2.77$ & & $6.48 \pm 2.42$ & & \\
\hline & Change & $0.87 \pm 1.50$ & & $2.48 \pm 2.25$ & & $0.002^{*}$ \\
\hline \multirow[t]{3}{*}{ Toilet } & Initial & $2.13 \pm 2.79$ & $<0.001^{*}$ & $1.94 \pm 2.53$ & 0.143 & \\
\hline & Final & $2.67 \pm 3.00$ & & $5.42 \pm 3.70$ & & \\
\hline & Change & $0.73 \pm 1.68$ & & $3.61 \pm 2.94$ & & $<0.001^{*}$ \\
\hline \multirow[t]{3}{*}{ Stair climbing } & Initial & $0.07 \pm 0.37$ & $0.001^{*}$ & $0.32 \pm 1.25$ & $0.039 *$ & \\
\hline & Final & $0.63 \pm 1.75$ & & $2.10 \pm 3.06$ & & \\
\hline & Change & $0.57 \pm 1.61$ & & $1.77 \pm 2.70$ & & $0.039 *$ \\
\hline \multirow[t]{3}{*}{ Dressing } & Initial & $3.03 \pm 2.44$ & $<0.001^{*}$ & $2.45 \pm 1.75$ & $0.027^{*}$ & \\
\hline & Final & $3.83 \pm 2.83$ & & $5.03 \pm 2.82$ & & \\
\hline & Change & $0.90 \pm 1.95$ & & $2.77 \pm 2.75$ & & $0.003^{*}$ \\
\hline \multirow[t]{3}{*}{ Bowel control } & Initial & $4.63 \pm 4.64$ & $0.002^{*}$ & $6.00 \pm 4.53$ & $0.011^{*}$ & \\
\hline & Final & $5.60 \pm 4.20$ & & $8.45 \pm 3.03$ & & \\
\hline & Change & $0.97 \pm 2.12$ & & $2.45 \pm 3.85$ & & 0.068 \\
\hline \multirow[t]{3}{*}{ Bladder control } & Initial & $4.83 \pm 4.77$ & $<0.001^{*}$ & $4.90 \pm 4.58$ & $0.017^{*}$ & \\
\hline & Final & $5.90 \pm 4.24$ & & $8.35 \pm 2.92$ & & \\
\hline & Change & $1.07 \pm 2.33$ & & $3.45 \pm 4.07$ & & $0.007^{*}$ \\
\hline \multirow[t]{3}{*}{ Ambulation or wheelchair } & Initial & $1.90 \pm 3.20$ & $<0.001^{*}$ & $1.32 \pm 3.40$ & $0.003^{*}$ & \\
\hline & Final & $3.73 \pm 4.51$ & & $4.10 \pm 4.71$ & & \\
\hline & Change & $1.83 \pm 3.13$ & & $2.97 \pm 3.71$ & & 0.203 \\
\hline \multirow[t]{3}{*}{ Transfer } & Initial & $4.87 \pm 3.95$ & $<0.001^{*}$ & $5.35 \pm 3.95$ & $0.002^{*}$ & \\
\hline & Final & $6.70 \pm 4.46$ & & $9.10 \pm 3.27$ & & \\
\hline & Change & $1.83 \pm 2.57$ & & $3.74 \pm 3.40$ & & $0.017^{*}$ \\
\hline
\end{tabular}

Values are presented as mean \pm standard deviation.

MBI, Modified Barthel Index; MoCA, Montreal Cognitive Assessment.

${ }^{*} \mathrm{p}<0.05$.

improvement was greater in the high MoCA score group than in the low MoCA group. In addition, a positive correlation was found between initial MoCA score and MBI improvement. As for the initial MMSE score and MBI improvement, no significant association was found.

Although improvement in the MMSE score was greater in the low MoCA group than in the high MoCA group, the MBI improvement was significantly greater in the high MoCA group than in the low MoCA group. This suggests that the ceiling effect is caused by the high initial MMSE $(20.51 \pm 4.58)$ of patients in the high MoCA group. Although MMSE is used as a routine examination for 
screening cognitive function in stroke patients, the majority of items in the MMSE are linked to orientation, which may undermine the accuracy in a patient's cognitive assessment. Also, MMSE results can be overestimated owing to the learning effects over repeated measurements. Therefore, a detailed neurological assessment test is needed to test for various cognitive dysfunctions to use early cognitive function as a predictor of functional outcomes in stroke patients [26].

MoCA has been developed as a more demanding test that includes evaluation of higher-level language, executive function, and complex visuospatial processing [27]. Executive function involves higher-order cognitive regulatory processes, including inhibitory control, working memory, and attention, which enhance a patient's capacity for problem-solving and sustained goal-oriented exploration [28]. Executive dysfunction is known to predict stroke risk, dementia, and mortality. Because executive functions can be tested by trails, clock drawing, and cube copying (5 points out of 30) in MoCA, it can detect more patients with executive dysfunction than the MMSE pentagon copying (1 point out of 30) [22]. Thus, MoCA has been found to detect executive dysfunction in stroke patients more sensitively than has MMSE $[16,29]$.

Many studies have utilized MoCA for assessing cognitive function related to the patient's functional impairment $[17,18]$. Schweizer et al. [30] reported that MoCA was more sensitive than MMSE in detecting cognitive impairment after hemorrhagic stroke. The ability of MoCA to better assess function in stroke patients than MMSE can be explained by the fact that the degree of functional disability in stroke patients is more evident in relation to attention and executive dysfunction than to memory deficits [31].

The group with higher initial MoCA scores demonstrated significantly greater improvement in functional impairment than the other group. The results remained consistent when other factors were controlled for (e.g., age, years of education).

In the high MoCA group, which is thought to have wellpreserved cognitive functions, including executive function, the score improvement was greater in most of the daily activity items related to executive function. This suggests that patients with higher MoCA can expect a significant functional improvement during initial rehabilitation, which is important for executive function [32].
Table 4. Linear regression of MoCA, MMSE as a predictor of functional outcome $(\mathrm{n}=61)$

\begin{tabular}{lcccc}
\hline & $\mathbf{B}$ & $\boldsymbol{\beta}$ & $\mathbf{R}^{2}$ & $\mathbf{p}$-value \\
\hline MBI at discharge & & & & \\
MoCA & 2.377 & 0.564 & 0.315 & $<0.001^{*}$ \\
MMSE & 2.067 & 0.619 & 0.372 & $<0.001^{*}$ \\
MBI improvement & & & & \\
MoCA & 1.427 & 0.407 & 0.241 & $<0.001^{*}$ \\
MMSE & 0.408 & 0.184 & 0.017 & 0.157 \\
\hline
\end{tabular}

MoCA, Montreal Cognitive Assessment; MMSE, MiniMental Status Examination; MBI, Modified Barthel Index. ${ }^{*} \mathrm{p}<0.05$.

On linear regression analysis, both initial MMSE and K-MoCA were positively correlated with follow-up MBI score. However, initial MMSE did not demonstrate a significant association with MBI improvement, while the initial K-MoCA score showed a significant positive correlation to MBI improvement (Table 4). This suggests that the MMSE score does not fully reflect the functional improvement. This is because many of the items in the MMSE are related to orientation, and many of the items in MoCA consist of evaluating executive function associated with functional improvement. According to a previous study, rehabilitation of executive function is related to functional improvement [32].

The significance of this study is that the improvement in functional impairment was correlated to the initial MoCA score and included patients with a wider range of functional performances and cognitive deficits from the Asian population. Initial assessment with MoCA may help predict the prognosis of subacute stroke patients. If the MoCA score remains relatively high even with severe functional impairment, one can expect considerable functional recovery after rehabilitation.

As this study had a retrospective design, a large number of patients were excluded; only subacute stroke patients were included in the study. So, future studies with a larger sample size are required to further validate our findings. At the time of discharge, we did not evaluate MoCA; hence, we could not assess the changes in MoCA scores between low MoCA and high MoCA group and the lesser ceiling effect in MoCA. Thus, future studies should include both initial and follow-up MoCA scores.

MoCA is more useful for assessing executive abilities and abstraction abilities. However, compared to MMSE, 
MoCA has more items involving reading and writing, resulting in low scores in patients who cannot read or write. Therefore, selection bias may have occurred because patients who cannot read or write were excluded when selecting patients.

In addition, after stroke, the functional outcome may be influenced not only by cognitive ability but also by other variables such as strength, language ability, and stiffness. So, future study will have to consider these various factors. We observed functional improvements in subacute stroke patients during short-term hospitalization; hence, we need to confirm the functional outcomes of patients through long-term studies.

In conclusion, we reviewed records of patients admitted to our department for rehabilitation after stroke. Our results indicated a significant positive correlation between the initial MoCA score and MBI improvement. Regarding the follow-up MBI score, both initial MMSE and MoCA were positively correlated to the follow-up MBI score. MoCA is a valuable option that can closely analyze executive dysfunction. An initial MoCA score may be able to predict functional improvement; higher initial MoCA scores, which reflect preservation of executive function, indicate better functional outcome in the subacute stroke phase.

\section{CONFLICT OF INTEREST}

No potential conflict of interest relevant to this article was reported.

\section{REFERENCES}

1. Walsh ME, Galvin R, Loughnane C, Macey C, Horgan NF. Community re-integration and long-term need in the first five years after stroke: results from a national survey. Disabil Rehabil 2015;37:1834-8.

2. Milinaviciene E, Rastenyte D, Krisciunas A. Effectiveness of the second-stage rehabilitation in stroke patients with cognitive impairment. Medicina (Kaunas) 2011;47:486-93.

3. Cioncoloni D, Piu P, Tassi R, Acampa M, Guideri F, Taddei S, et al. Relationship between the modified Rankin Scale and the Barthel Index in the process of functional recovery after stroke. NeuroRehabilitation 2012;30:315-22.
4. Hankey GJ, Jamrozik K, Broadhurst RJ, Forbes S, Anderson CS. Long-term disability after first-ever stroke and related prognostic factors in the Perth Community Stroke Study, 1989-1990. Stroke 2002;33:1034-40.

5. Hill RD, Backman L, Fratiglioni L. Determinants of functional abilities in dementia. J Am Geriatr Soc 1995;43:1092-7.

6. Tatemichi TK, Desmond DW, Stern Y, Paik M, Sano M, Bagiella E. Cognitive impairment after stroke: frequency, patterns, and relationship to functional abilities. J Neurol Neurosurg Psychiatry 1994;57:202-7.

7. Nys GM, van Zandvoort MJ, de Kort PL, Jansen BP, de Haan EH, Kappelle LJ. Cognitive disorders in acute stroke: prevalence and clinical determinants. Cerebrovasc Dis 2007;23:408-16.

8. Rasquin SM, Lodder J, Ponds RW, Winkens I, Jolles J, Verhey FR. Cognitive functioning after stroke: a oneyear follow-up study. Dement Geriatr Cogn Disord 2004;18:138-44.

9. Sachdev PS, Brodaty H, Valenzuela MJ, Lorentz LM, Koschera A. Progression of cognitive impairment in stroke patients. Neurology 2004;63:1618-23.

10. Galski T, Bruno RL, Zorowitz R, Walker J. Predicting length of stay, functional outcome, and aftercare in the rehabilitation of stroke patients: the dominant role of higher-order cognition. Stroke 1993;24:1794800 .

11. Larson EB, Kirschner K, Bode RK, Heinemann AW, Clorfene J, Goodman R. Brief cognitive assessment and prediction of functional outcome in stroke. Top Stroke Rehabil 2003;9:10-21.

12. Paker N, Bugdayci D, Tekdos D, Kaya B, Dere C. Impact of cognitive impairment on functional outcome in stroke. Stroke Res Treat 2010;2010:652612.

13. Ozdemir F, Birtane M, Tabatabaei R, Ekuklu G, Kokino S. Cognitive evaluation and functional outcome after stroke. Am J Phys Med Rehabil 2001;80:410-5.

14. Nys GM, van Zandvoort MJ, de Kort PL, Jansen BP, Kappelle LJ, de Haan EH. Restrictions of the MiniMental State Examination in acute stroke. Arch Clin Neuropsychol 2005;20:623-9.

15. Tombaugh TN, McIntyre NJ. The mini-mental state examination: a comprehensive review. J Am Geriatr Soc 1992;40:922-35.

16. Dong Y, Sharma VK, Chan BP, Venketasubramanian N, Teoh HL, Seet RC, et al. The Montreal Cognitive As- 
sessment (MoCA) is superior to the Mini-Mental State Examination (MMSE) for the detection of vascular cognitive impairment after acute stroke. J Neurol Sci 2010;299:15-8.

17. Durant J, Leger GC, Banks SJ, Miller JB. Relationship between the activities of daily living questionnaire and the Montreal cognitive assessment. Alzheimers Dement (Amst) 2016;4:43-6.

18. Toglia J, Fitzgerald KA, O’Dell MW, Mastrogiovanni AR, Lin CD. The Mini-Mental State Examination and Montreal Cognitive Assessment in persons with mild subacute stroke: relationship to functional outcome. Arch Phys Med Rehabil 2011;92:792-8.

19. Chui HC, Victoroff JI, Margolin D, Jagust W, Shankle R, Katzman R. Criteria for the diagnosis of ischemic vascular dementia proposed by the State of California Alzheimer's Disease Diagnostic and Treatment Centers. Neurology 1992;42(3 Pt 1):473-80.

20. Reisberg B, Franssen EH, Souren LE, Auer S, Kenowsky S. Progression of Alzheimer's disease: variability and consistency: ontogenic models, their applicability and relevance. J Neural Transm Suppl 1998; 54:9-20.

21. Kazui H, Yoshiyama K, Kanemoto H, Suzuki Y, Sato S, Hashimoto M, et al. Differences of behavioral and psychological symptoms of dementia in disease severity in four major dementias. PLoS One 2016;11:e0161092.

22. Mai LM, Sposato LA, Rothwell PM, Hachinski V, Pendlebury ST. A comparison between the MoCA and the MMSE visuoexecutive sub-tests in detecting abnormalities in TIA/stroke patients. Int J Stroke 2016; 11:420-4.

23. Kang YW, Park JS, Yu KH, Lee BC. A reliability, validity, and normative study of the Korean-Montreal Cognitive Assessment (K-MoCA) as an instrument for screening of Vascular Cognitive Impairment (VCI). Korean J Clin Psychol 2009;28:549-62.

24. Folstein MF, Folstein SE, McHugh PR. "Mini-mental state". A practical method for grading the cognitive state of patients for the clinician. J Psychiatr Res 1975;
12:189-98.

25. Bergeron D, Flynn K, Verret L, Poulin S, Bouchard RW, Bocti C, et al. Multicenter validation of an MMSE-MoCA conversion table. J Am Geriatr Soc 2017;65:106772.

26. Park J, Lee G, Lee SU, Jung SH. The impact of acute phase domain-specific cognitive function on poststroke functional recovery. Ann Rehabil Med 2016;40: 214-22.

27. Nasreddine ZS, Phillips NA, Bedirian V, Charbonneau S, Whitehead V, Collin I, et al. The Montreal Cognitive Assessment, MoCA: a brief screening tool for mild cognitive impairment. J Am Geriatr Soc 2005;53:6959.

28. Carlson SM. Developmentally sensitive measures of executive function in preschool children. Dev Neuropsychol 2005;28:595-616.

29. Pendlebury ST, Cuthbertson FC, Welch SJ, Mehta Z, Rothwell PM. Underestimation of cognitive impairment by Mini-Mental State Examination versus the Montreal Cognitive Assessment in patients with transient ischemic attack and stroke: a population-based study. Stroke 2010;41:1290-3.

30. Schweizer TA, Al-Khindi T, Macdonald RL. MiniMental State Examination versus Montreal Cognitive Assessment: rapid assessment tools for cognitive and functional outcome after aneurysmal subarachnoid hemorrhage. J Neurol Sci 2012;316:137-40.

31. Stephens S, Kenny RA, Rowan E, Kalaria RN, Bradbury M, Pearce R, et al. Association between mild vascular cognitive impairment and impaired activities of daily living in older stroke survivors without dementia. J Am Geriatr Soc 2005;53:103-7.

32. Vizzotto AD, Celestino DL, Buchain PC, Oliveira AM, Oliveira GM, Di Sarno ES, et al. A pilot randomized controlled trial of the Occupational Goal Intervention method for the improvement of executive functioning in patients with treatment-resistant schizophrenia. Psychiatry Res 2016;245:148-56. 